\title{
ON A PLANAR VARIANT OF THE KAKEYA PROBLEM
}

\author{
KeITH M. Rogers
}

\begin{abstract}
A $\mathcal{K}_{2}^{n}$-set is a set of zero Lebesgue measure containing a translate of every plane in an $(n-2)$-dimensional manifold in $\operatorname{Gr}(n, 2)$, where the manifold fulfills a curvature condition. We show that this is a natural class of sets with respect to the Kakeya problem and prove that $\operatorname{dim}_{H}(E) \geq 7 / 2$ for all $\mathcal{K}_{2}^{4}$-sets $E$. When the underlying field is replaced by $\mathbb{C}$, we get $\operatorname{dim}_{H}(E) \geq 7$ for all $\mathcal{K}_{2}^{4}$-sets over $\mathbb{C}$, and we construct an example to show that this is sharp. Thus $\mathcal{K}_{2}^{4}$-sets over $\mathbb{C}$ do not necessarily have full Hausdorff dimension.
\end{abstract}

\section{Introduction}

A Besicovitch set (with lines) in $\mathbb{R}^{n}$ is a set of zero Lebesgue measure that contains a translate of every member of $\operatorname{Gr}(n, 1)$, where $\operatorname{Gr}(n, 1)$ is the Grassmanian manifold of 1 -dimensional linear subspaces of $\mathbb{R}^{n}$.

A construction of A. Besicovitch [1] led to the surprising fact that such sets exist. The Kakeya conjecture asserts that Besicovitch sets must have full Hausdorff dimension.

We note that $\operatorname{Gr}(n, 1)$ has dimension $n-1$ and a line has dimension 1 , and the union of these lines will fill the space and have dimension $n$. Informally, the Kakeya conjecture asserts that under translation of the lines, the dimensions continue to add up, or that the intersection remains negligible.

The conjecture has been solved in the affirmative in the plane, but is open for $n \geq 3$. T. Wolff [14] proved that $\operatorname{dim}_{H}(E) \geq(n+2) / 2$ for all Besicovitch sets $E$ in $\mathbb{R}^{n}$, where $\operatorname{dim}_{H}$ denotes Hausdorff dimension, and there has been much recent progress for higher dimensions (see [3], [5], [7], [8], [11]).

N. H. Katz, I. Łaba and T. Tao [6] have shown that the Minkowski dimension of a Kakeya set in $\mathbb{R}^{3}$ is strictly greater than $5 / 2$. They also show that if a Kakeya set in $\mathbb{R}^{3}$ has dimension close to $5 / 2$, then it must exhibit a certain structural property that they call 'planiness'. Roughly speaking, most of the lines that pass through a point in the Besicovitch set, lie in the union of a small number of planes. It seems reasonable then, to consider the variant of the problem where lines are replaced by planes.

An $(n, 2)$-set is a set that contains a translate of every member of $\operatorname{Gr}(n, 2)$, where $\operatorname{Gr}(n, 2)$ is the Grassmanian manifold of 2-dimensional linear subspaces

Received by the editors June 6, 2005.

2000 Mathematics Subject Classification. 42B10, $28 \mathrm{~A} 75$.

Key words and phrases. Kakeya, planes, curvature.

Supported by the EC project HPRN-CT-2001-00273 - HARP. 
of $\mathbb{R}^{n}$. J. Bourgain [2] proved that $(4,2)$-sets have strictly positive Lebesgue measure, so that there are no, so called, Besicovitch $(4,2)$-sets. Similarly, there are no Besicovitch $(3,2)$-sets. T. Mitsis [9] recently claimed that $(n, 2)$-sets have full Hausdorff dimension when $n \geq 5$, although unfortunately the argument is incomplete.

As $\operatorname{Gr}(n, 2)$ has dimension $2(n-2)$ and a plane has dimension 2, these results do not have the same informal interpretation as that of the Kakeya problem. Indeed the planes of an $(n, 2)$-set will inevitably have, in some sense, nontrivial intersection.

Initially, it appears reasonable to ask whether sets containing translates of an $(n-2)$-dimensional manifold in $\operatorname{Gr}(n, 2)$ necessarily have full Hausdorff dimension. Without further restriction on the manifold however, this will fail. For instance, a 2-dimensional manifold in $\operatorname{Gr}(4,2)$ could have all of its planes contained in a 3-space, so that the set consisting of the union of these planes would have Hausdorff dimension 3. We outlaw this by adding a curvature condition which is essentially a version of the Wolff axiom ([6], [10], [14]) for planes.

Let $\pi, \pi^{\prime} \in \operatorname{Gr}(n, 2)$, and define the major angle between $\pi$ and $\pi^{\prime}$ by

$$
\theta\left(\pi, \pi^{\prime}\right)=\left\|\operatorname{proj}_{\pi}-\operatorname{proj}_{\pi^{\prime}}\right\|,
$$

where $\operatorname{proj}_{\pi}: \mathbb{R}^{n} \rightarrow \pi$ is the orthogonal projection onto $\pi$. We note that $\theta$ takes values from zero to one, so this is not a standard angle. As $\theta$ is a metric, we can induce a measure $\mu$ on the manifold $\mathcal{M} \subset \operatorname{Gr}(n, 2)$, which we normalise to have total mass one. We denote by $B_{\epsilon}(\pi)$ the ball

$$
\left\{\pi^{\prime} \in \operatorname{Gr}(n, 2): \theta\left(\pi, \pi^{\prime}\right)<\epsilon\right\},
$$

and denote by $\mathcal{M}_{\Lambda_{m}}^{\epsilon}$ the set

$$
\left\{\pi^{\prime} \in \mathcal{M}: \min _{\pi \subset \Lambda_{m}} \theta\left(\pi, \pi^{\prime}\right)<\epsilon\right\},
$$

where $\Lambda_{m} \subset \mathbb{R}^{n}$ is an $m$-space with $3 \leq m \leq n-1$.

An $(n-2)$-dimensional manifold $\mathcal{M} \subset \operatorname{Gr}(n, 2)$ is said to be curved if there exists a constant $C$ such that

$$
\frac{\mu\left(\mathcal{M}_{\Lambda_{m}}^{\epsilon_{1}} \cap B_{\epsilon_{2}}(\pi)\right)}{\epsilon_{1}^{m-2} \epsilon_{2}^{n-m}}<C
$$

for all $\epsilon_{1}, \epsilon_{2}>0, m$-spaces $\Lambda_{m} \subset \mathbb{R}^{n}$ and $\pi \in \mathcal{M}$.

The condition forces the dimension of $\left\{\pi \in \mathcal{M}: \pi \subset \Lambda_{m}\right\}$ to be less than or equal to $m-2$ for all $m$-spaces $\Lambda_{m}$, and the manifold to be more evenly distributed in $\operatorname{Gr}(n, 2)$. The reason that this is known as an axiom in the Kakeya problem, is that the corresponding condition is automatically fulfilled by $\operatorname{Gr}(n, 1)$. This is not the case for planes, so we are obliged to include it in our definition.

A $\mathcal{K}_{2}^{n}$-set is a set of zero Lebesgue measure that contains a translate of every member of an $(n-2)$-dimensional curved manifold $\mathcal{M} \subset \operatorname{Gr}(n, 2)$.

As $\{l \times \mathbb{R}: l \in \operatorname{Gr}(n-1,1)\}$ is an $(n-2)$-dimensional curved manifold, a Besicovitch set that is constructed by taking the cross product of a Besicovitch 
set in $\mathbb{R}^{n-1}$ with a copy of $\mathbb{R}$ is also a $\mathcal{K}_{2}^{n}$-set. Thus we have the existence of such sets.

In Section 4 we will prove the following results.

Theorem 1. Let $E$ be a $\mathcal{K}_{2}^{3}$-set. Then $\operatorname{dim}_{H}(E)=3$.

Thus $\mathcal{K}_{2}^{3}$-sets have full Hausdorff dimension. Our main concern will be the proof of the following result.

Theorem 2. Let $E$ be a $\mathcal{K}_{2}^{4}$-set. Then $\operatorname{dim}_{H}(E) \geq 7 / 2$.

If we replace the underlying field by $\mathbb{C}$ and multiply the exponents of $\epsilon_{1}$ and $\epsilon_{2}$ in (1) by two, then the proof of Theorem 2 can be modified to obtain the following bound. The modification involves little more than changing the relevant exponents.

Theorem 3. Let $E$ be a $\mathcal{K}_{2}^{4}$-set over $\mathbb{C}$. Then $\operatorname{dim}_{H}(E) \geq 7$.

In Section 5, we will construct an example to show that Theorem 3 is sharp. Thus $\mathcal{K}_{2}^{4}$-sets over $\mathbb{C}$ do not necessarily have full Hausdorff dimension.

\section{Geometric preliminaries}

Throughout $\delta$ will be a real parameter such that $0<\delta \ll 1$, and $\pi$ will denote a plane, and never the usual number. We say that a set $\Pi \subset \operatorname{Gr}(n, 2)$ is $\delta$-separated if $\theta\left(\pi, \pi^{\prime}\right)>\delta$ for all $\pi, \pi^{\prime} \in \Pi$.

We use $A \lesssim B$ to denote the estimate $A \leq C_{\epsilon} \delta^{-\epsilon} B$ for all $\epsilon>0$, where $C_{\epsilon}$ is a constant depending only on $\epsilon$ and the manifold $\mathcal{M}$. This notation will be convenient, as factors of $\log (1 / \delta)$ will simply disappear. We use $A \sim B$ to denote $B / 2<A \leq B$.

We will require another notion of an angle between two planes. The need for two notions is created by the fact that in $\mathbb{R}^{n}$, where $n \geq 4$, the intersection of two planes can be a point, and not necessarily a line. Let $l, l^{\prime} \in \operatorname{Gr}(n, 1)$, and define the minor angle between $\pi$ and $\pi^{\prime}$ by

$$
\underline{\theta}\left(\pi, \pi^{\prime}\right)=\min _{l \subset \pi, l^{\prime} \subset \pi^{\prime}}\left\|\operatorname{proj}_{l}-\operatorname{proj}_{l^{\prime}}\right\| .
$$

Informally, $\underline{\theta}$ can be considered to be the smallest angle between two planes. If $\pi, \pi^{\prime} \in \operatorname{Gr}(n, 2)$ intersect in a line, then $\underline{\theta}\left(\pi, \pi^{\prime}\right)=0$, so that $\underline{\theta}$ is not a metric.

Define a plate $P_{\pi}$ to be the image of $[0,1] \times[0,1] \times \underbrace{[0, \delta] \times \cdots \times[0, \delta]}_{n-2}$ under a rotation and translation, such that its face of area one is parallel to $\pi \in \operatorname{Gr}(n, 2)$. Define $S_{\pi}$ to be the central unit square of the plate $P_{\pi}$. When $P_{\pi} \cap P_{\pi^{\prime}} \neq \emptyset$ and $\theta\left(\pi, \pi^{\prime}\right)=\phi$, we say the plates intersect at major angle $\phi$. Similarly, when $P_{\pi} \cap P_{\pi^{\prime}} \neq \emptyset$ and $\underline{\theta}\left(\pi, \pi^{\prime}\right)=\phi$, we say the plates intersect at minor angle $\phi$.

We will require the following lemmas. The first is due to Mitsis [9] and is a natural extension of an observation of A. Córdoba [4]. We include the proof for convenience. The third is a natural extension of an observation of Wolff [14]. 
Lemma 4. Let $\pi_{1}, \pi_{2} \in \operatorname{Gr}(n, 2)$. Then

$$
\left|P_{\pi_{1}} \cap P_{\pi_{2}}\right| \lesssim \frac{\delta^{n-1}}{\theta\left(\pi_{1}, \pi_{2}\right)} .
$$

Proof. We have that $P_{\pi_{1}} \cap P_{\pi_{2}}$ is contained in $\left(R_{1} \cap R_{2}\right) \times R_{3}$, where $R_{1}$ and $R_{2}$ are 2-dimensional rectangles of dimension $1 \times \delta$, and $R_{3}$ is an $(n-2)$-dimensional cuboid of dimension $1 \times \underbrace{\delta \times \cdots \times \delta}_{n-3}$. The rectangles $R_{1}$ and $R_{2}$ intersect at an angle $\gtrsim \theta\left(\pi_{1}, \pi_{2}\right)$, so by elementary geometry,

$$
\mathcal{L}^{2}\left(R_{1} \cap R_{2}\right) \lesssim \frac{\delta^{2}}{\theta\left(\pi_{1}, \pi_{2}\right)},
$$

and the lemma follows.

Lemma 5. Let $\pi_{1}, \pi_{2} \in \operatorname{Gr}(n, 2)$. Then

$$
\left|P_{\pi_{1}} \cap P_{\pi_{2}}\right| \lesssim \frac{\delta^{n}}{\theta\left(\pi_{1}, \pi_{2}\right) \underline{\theta}\left(\pi_{1}, \pi_{2}\right)} .
$$

Proof. By translation we can suppose that the origin is contained in $P_{\pi_{1}} \cap P_{\pi_{2}}$. Let $x \in P_{\pi_{1}} \cap P_{\pi_{2}}$ and define $l \in \operatorname{Gr}(n, 1)$ to be the line that passes through the origin and $x$. Define $l_{1}, l_{2} \in \operatorname{Gr}(n, 1)$ to be the orthogonal projections of $l$ onto $\pi_{1}$ and $\pi_{2}$ respectively. Now by elementary geometry,

$$
\left\|\operatorname{proj}_{l}-\operatorname{proj}_{l_{1}}\right\| \lesssim \delta /|x|
$$

and

$$
\left\|\operatorname{proj}_{l}-\operatorname{proj}_{l_{2}}\right\| \lesssim \delta /|x|
$$

By the triangle inequality,

$$
\underline{\theta}\left(\pi_{1}, \pi_{2}\right) \leq\left\|\operatorname{proj}_{l_{1}}-\operatorname{proj}_{l_{2}}\right\| \lesssim \delta /|x|,
$$

so that

$$
|x| \lesssim \delta / \underline{\theta}\left(\pi_{1}, \pi_{2}\right) .
$$

If we denote the diameter of $P_{\pi_{1}} \cap P_{\pi_{2}}$ by $\alpha$, then we see that $\alpha \lesssim \delta / \underline{\theta}\left(\pi_{1}, \pi_{2}\right)$.

As in the previous proof, $P_{\pi_{1}} \cap P_{\pi_{2}}$ is contained in $\left(R_{1} \cap R_{2}\right) \times R_{3}$, where $R_{1}$ and $R_{2}$ are 2-dimensional rectangles of dimension $1 \times \delta$, and $R_{3}$ is an $(n-2)-$ dimensional cuboid of dimension $\alpha \times \underbrace{\delta \times \cdots \times \delta}_{n-3}$. We are able to reduce the length of the long side of $R_{3}$ as we have a bound on the diameter of $P_{\pi_{1}} \cap P_{\pi_{2}}$.

The rectangles $R_{1}$ and $R_{2}$ intersect at an angle $\gtrsim \theta\left(\pi, \pi^{\prime}\right)$, so by elementary geometry,

$$
\mathcal{L}^{2}\left(R_{1} \cap R_{2}\right) \lesssim \frac{\delta^{2}}{\theta\left(\pi, \pi^{\prime}\right)}
$$

and the lemma follows.

Finally we prove a quantitative version of the fact that three planes intersecting in distinct lines are contained in a $3-$ plane. 
Lemma 6. Let $\pi_{0}, \pi_{1}, \pi_{2} \in \operatorname{Gr}(n, 2)$, and define $\Sigma=\left\{x \in \mathbb{R}^{n}: d\left(x, S_{\pi_{0}}\right)>\nu\right\}$, where $\delta<\nu<1$. Suppose that $P_{\pi_{1}}, P_{\pi_{2}}$ intersect $P_{\pi_{0}}$ at major angles $\sim 1$ and minor angles $<\phi$, where $\delta \leq \phi \leq 1$, and suppose that

$$
P_{\pi_{1}} \cap P_{\pi_{2}} \cap \Sigma \neq \emptyset .
$$

Then there is a 3-space $\Lambda$, chosen independently of $\pi_{2}$, such that

$$
\min _{\pi \subset \Lambda} \theta\left(\pi, \pi_{1}\right)<\phi \quad \text { and } \quad \min _{\pi \subset \Lambda} \theta\left(\pi, \pi_{2}\right) \lesssim \phi / \nu .
$$

Proof. By translation we can suppose that the origin is contained in the set

$$
\left\{x: d\left(x, P_{\pi_{0}} \cap P_{\pi_{1}}\right)<\delta\right\} \cap S_{\pi_{0}},
$$

where $d(x, A)=\inf _{y \in A}|x-y|$. Now as $\underline{\theta}\left(\pi_{0}, \pi_{1}\right)<\phi$, there are lines $l_{0}, l_{1} \in$ $\operatorname{Gr}(n, 1)$ that are contained in $\pi_{0}$ and $\pi_{1}$ respectively, such that

$$
\left\|\operatorname{proj}_{l_{0}}-\operatorname{proj}_{l_{1}}\right\|<\phi .
$$

Let $l_{1}^{\prime} \in \operatorname{Gr}(n, 1)$ be the line contained in $\pi_{1}$ that is orthogonal to $l_{1}$, and define $\Lambda$ to be the 3 -space spanned by $\pi_{0}$ and $l_{1}^{\prime}$.

Define $\pi_{1}^{\prime} \in \operatorname{Gr}(n, 2)$ to be the plane spanned by $l_{0}$ and $l_{1}^{\prime}$, so that $\pi_{1}^{\prime}$ is contained in $\Lambda$. Now by elementary geometry, we have $\theta\left(\pi_{1}, \pi_{1}^{\prime}\right)<\phi$ and

$$
\min _{\pi \subset \Lambda} \theta\left(\pi, \pi_{1}\right)<\phi .
$$

Define $T: \mathbb{R}^{n} \rightarrow \mathbb{R}^{n}$ to be a translation that maps a point in

$$
\left\{x: d\left(x, P_{\pi_{0}} \cap P_{\pi_{2}}\right)<\delta\right\} \cap S_{\pi_{0}}
$$

to the origin. We note that $\Lambda$ and $\Sigma$ are essentially invariant under the action of $T$, so that if $\zeta \in P_{\pi_{1}} \cap P_{\pi_{2}} \cap \Sigma$, then there exists a $\zeta^{\prime} \in \Lambda \cap \Sigma$ such that $\left|T(\zeta)-\zeta^{\prime}\right| \lesssim \phi$

Now as $\underline{\theta}\left(\pi_{0}, \pi_{2}\right)<\phi$, there are lines $l_{0}^{\prime}, l_{2} \in \operatorname{Gr}(n, 1)$ that are contained in $\pi_{0}$ and $\pi_{2}$ respectively, such that

$$
\left\|\operatorname{proj}_{l_{0}^{\prime}}-\operatorname{proj}_{l_{2}}\right\|<\phi .
$$

Define $\pi_{2}^{\prime} \in \operatorname{Gr}(n, 2)$ to be the plane that contains $l_{0}^{\prime}$ and $\zeta^{\prime}$, so that $\pi_{2}^{\prime}$ is contained in $\Lambda$. Now by elementary geometry, we have $\theta\left(\pi_{2}, \pi_{2}^{\prime}\right) \lesssim \phi / \nu$ and

$$
\min _{\pi \subset \Lambda} \theta\left(\pi, \pi_{2}\right) \lesssim \phi / \nu
$$

as required.

\section{The main argument}

The (concave) triangle inequality states that when $p \leq 1$,

$$
\left\|\sum_{k=0}^{N} f_{k}\right\|_{p}^{p} \leq \sum_{k=0}^{N}\left\|f_{k}\right\|_{p}^{p}
$$


and this will frequently take the following form: If $\left\|f_{k}\right\|_{p}^{p} \lesssim C$ for all $k$, then

$$
\left\|\sum_{k=0}^{\left\lfloor\log _{2}(1 / \delta)\right\rfloor} f_{k}\right\|_{p}^{p} \lesssim C .
$$

Similarly, the pigeonhole principle will often take the form: If

$$
\sum_{k=0}^{\left\lfloor\log _{2}(1 / \delta)\right\rfloor}\left\|f_{k}\right\| \gtrsim C,
$$

then for some $k$, we have $\left\|f_{k}\right\| \gtrsim C$.

The following lemma will be key to the proof of Theorem 1 .

Lemma 7. Let $\Pi$ be a $\delta$-separated subset of a 1-dimensional manifold $\mathcal{M}$ in $\operatorname{Gr}(3,2)$. Then

$$
\left\|\sum_{\pi \in \Pi} \chi_{P_{\pi}}\right\|_{2} \lesssim 1
$$

Proof. We note that as

$$
\left\|\sum_{\pi \in \Pi} \chi_{P_{\pi}}\right\|_{2}^{2}=\left\|\sum_{\pi \in \Pi} \sum_{\pi^{\prime} \in \Pi} \chi_{P_{\pi}} \chi_{P_{\pi^{\prime}}}\right\|_{1},
$$

it will suffice to show

$$
\left\|\sum_{\pi \in \Pi} \sum_{\pi^{\prime} \in \Pi} \chi_{P_{\pi}} \chi_{P_{\pi^{\prime}}}\right\|_{1} \lesssim 1
$$

Now

$$
\sum_{\pi \in \Pi} \sum_{\pi^{\prime} \in \Pi} \chi_{P_{\pi}} \chi_{P_{\pi^{\prime}}} \leq \sum_{k=0}^{\left\lfloor\log _{2}(1 / \delta)\right\rfloor} \sum_{\pi, \pi^{\prime}: \theta\left(\pi, \pi^{\prime}\right) \sim 2^{-k}} \chi_{P_{\pi}} \chi_{P_{\pi^{\prime}}}+\sum_{\pi \in \Pi} \chi_{P_{\pi}},
$$

as $\Pi$ is $\delta$-separated. Thus, by the triangle inequality, it will suffice to show

$$
\left\|\sum_{\pi, \pi^{\prime}: \theta\left(\pi, \pi^{\prime}\right) \sim 2^{-k}} \chi_{P_{\pi}} \chi_{P_{\pi^{\prime}}}\right\|_{1} \lesssim 1
$$

for all $k$ less than or equal to $\left\lfloor\log _{2}(1 / \delta)\right\rfloor$, and

$$
\left\|\sum_{\pi \in \Pi} \chi_{P_{\pi}}\right\|_{1} \lesssim 1
$$

Again, by the triangle inequality,

$$
\left\|\sum_{\pi \in \Pi} \chi_{P_{\pi}}\right\|_{1} \leq \sum_{\pi \in \Pi}\left|P_{\pi}\right| \lesssim 1,
$$

as $\# \Pi \lesssim \delta^{-1}$ and $\left|P_{\pi}\right|=\delta$. Thus it remains to show (2) for each $k$, which we now consider to be fixed.

Using the metric $\theta$ on the 1 -dimensional manifold $\mathcal{M}$, we can cover $\Pi$ by a constant multiple of $2^{k}$ balls $\left\{B_{j}\right\}$, with radius a constant multiple of $2^{-k}$. 
We can also choose the cover so that if $\theta\left(\pi, \pi^{\prime}\right) \sim 2^{-k}$, then $\pi$ and $\pi^{\prime}$ are both contained in some $B_{j}$. Hence, by the triangle inequality, it will suffice to prove

$$
\left\|\sum_{\substack{\pi, \pi^{\prime} \in B_{j} \cap \Pi \\ \theta\left(\pi, \pi^{\prime}\right) \sim 2^{-k}}} \chi_{P_{\pi}} \chi_{P_{\pi^{\prime}}}\right\|_{1} \lesssim 2^{-k}
$$

for all $k$ less than or equal to $\left\lfloor\log _{2}(1 / \delta)\right\rfloor$, and each ball $B_{j}$.

Without loss of generality, we can suppose that $B_{j}$ is centered on the $x_{1} x_{2}-$ plane. Define the dilation $L: \mathbb{R}^{3} \rightarrow \mathbb{R}^{3}$ by

$$
L\left(x_{1}, x_{2}, x_{3}\right)=\left(x_{1}, x_{2}, 2^{k} x_{3}\right) .
$$

We scale our geometric configuration by $L$, so that

$$
\left\|\sum_{\substack{\pi, \pi^{\prime} \in B_{j} \cap \Pi \\ \theta\left(\pi, \pi^{\prime}\right) \sim 2^{-k}}} \chi_{P_{\pi}} \chi_{P_{\pi^{\prime}}}\right\|_{1}=2^{-k}\left\|\sum_{\substack{\pi, \pi^{\prime} \in B_{j} \cap \Pi \\ \theta\left(\pi, \pi^{\prime}\right) \sim 2^{-k}}} \chi_{L\left(P_{\pi}\right)} \chi_{L\left(P_{\pi^{\prime}}\right)}\right\|_{1} .
$$

Now as we have essentially changed $\delta$ to $2^{k} \delta$, and $\theta\left(L(\pi), L\left(\pi^{\prime}\right)\right) \sim 1$, if we can prove (3) when $k=0$, then

$$
\left\|\sum_{\substack{\pi, \pi^{\prime} \in B_{j} \cap \Pi \\ \theta\left(\pi, \pi^{\prime}\right) \sim 2^{-k}}} \chi_{L\left(P_{\pi}\right)} \chi_{L\left(P_{\pi^{\prime}}\right)}\right\|_{1} \leq C_{\epsilon}\left(2^{k} \delta\right)^{-\epsilon}<C_{\epsilon} \delta^{-\epsilon} \lesssim 1,
$$

so that by (4),

$$
\left\|\sum_{\substack{\pi, \pi^{\prime} \in B_{j} \cap \Pi \\ \theta\left(\pi, \pi^{\prime}\right) \sim 2^{-k}}} \chi_{P_{\pi}} \chi_{P_{\pi^{\prime}}}\right\|_{1} \lesssim 2^{-k} .
$$

Thus it will suffice to prove (3) when $k=0$.

Now

$$
\left\|\sum_{\theta\left(\pi, \pi^{\prime}\right) \sim 1} \chi_{P_{\pi}} \chi_{P_{\pi^{\prime}}}\right\|_{1} \leq \sum_{\theta\left(\pi, \pi^{\prime}\right) \sim 1}\left|P_{\pi} \cap P_{\pi^{\prime}}\right| \lesssim(\# \Pi)^{2} \delta^{2} \lesssim 1,
$$

by Lemma 4, and we are done.

The following lemma will be key to the proof of Theorem 2 .

Lemma 8. Let $\Pi$ be a $\delta$-separated subset of a 2-dimensional curved manifold $\mathcal{M}$ in $\operatorname{Gr}(4,2)$. Then

$$
\left\|\sum_{\pi \in \Pi} \chi_{P_{\pi}}\right\|_{5 / 3} \lesssim \delta^{-1 / 5}
$$

Proof. The proof is based on the ideas of Wolff [14]. The key geometric fact used there, is that three lines intersecting in distinct points are contained in a plane. The corresponding fact here, is that three planes intersecting in distinct lines are contained in a 3-plane. Unfortunately, the intersection between two planes can be a point as well as a line.

In order to deal with the different types of intersection, we use the bilinear reduction of T. Tao, A. Vargas and L. Vega [13], which can also be found in [12]. This enables us to quantify, using the minor angle, how near the planes are to 
intersecting in lines. When the planes are not intersecting in lines, we are able to use Lemma 5, in place of Lemma 4, in compensation.

We make the bilinear reduction. Essentially this means we will begin by attempting to copy the proof of Lemma 7 . We note that as

$$
\left\|\sum_{\pi \in \Pi} \chi_{P_{\pi}}\right\|_{5 / 3}^{5 / 3}=\left\|\sum_{\pi \in \Pi} \sum_{\pi^{\prime} \in \Pi} \chi_{P_{\pi}} \chi_{P_{\pi^{\prime}}}\right\|_{5 / 6}^{5 / 6},
$$

it will suffice to show

$$
\left\|\sum_{\pi \in \Pi} \sum_{\pi^{\prime} \in \Pi} \chi_{P_{\pi}} \chi_{P_{\pi^{\prime}}}\right\|_{5 / 6}^{5 / 6} \lesssim \delta^{-1 / 3}
$$

Now

$$
\sum_{\pi \in \Pi} \sum_{\pi^{\prime} \in \Pi} \chi_{P_{\pi}} \chi_{P_{\pi^{\prime}}} \leq \sum_{k=0}^{\left\lfloor\log _{2}(1 / \delta)\right\rfloor} \sum_{\pi, \pi^{\prime}: \theta\left(\pi, \pi^{\prime}\right) \sim 2^{-k}} \chi_{P_{\pi}} \chi_{P_{\pi^{\prime}}}+\sum_{\pi \in \Pi} \chi_{P_{\pi}},
$$

as $\Pi$ is $\delta$-separated. Thus, by the triangle inequality, it will suffice to show

$$
\left\|\sum_{\pi, \pi^{\prime}: \theta\left(\pi, \pi^{\prime}\right) \sim 2^{-k}} \chi_{P_{\pi}} \chi_{P_{\pi^{\prime}}}\right\|_{5 / 6}^{5 / 6} \lesssim \delta^{-1 / 3}
$$

for all $k$ less than or equal to $\left\lfloor\log _{2}(1 / \delta)\right\rfloor$, and

$$
\left\|\sum_{\pi \in \Pi} \chi_{P_{\pi}}\right\|_{5 / 6}^{5 / 6} \lesssim \delta^{-1 / 3}
$$

Again by the triangle inequality,

$$
\left\|\sum_{\pi \in \Pi} \chi_{P_{\pi}}\right\|_{5 / 6}^{5 / 6} \leq\left\|\sum_{\pi \in \Pi} \chi_{P_{\pi}}\right\|_{1} \leq \sum_{\pi \in \Pi}\left|P_{\pi}\right| \lesssim 1<\delta^{-1 / 3}
$$

as $\# \Pi \lesssim \delta^{-2}$ and $\left|P_{\pi}\right|=\delta^{2}$, so it remains to show (5) for each $k$, which we now consider to be fixed.

Using the metric $\theta$ on the 2 -dimensional manifold $\mathcal{M}$, we can cover $\Pi$ by a constant multiple of $2^{2 k}$ balls $\left\{B_{j}\right\}$, with radius a constant multiple of $2^{-k}$. We can also choose the cover so that if $\theta\left(\pi, \pi^{\prime}\right) \leq 2^{-k}$, then $\pi$ and $\pi^{\prime}$ are both contained in some $B_{j}$. Hence, by the triangle inequality, it will suffice to prove

$$
\left\|\sum_{\substack{\pi, \pi^{\prime} \in B_{j} \cap \Pi \\ \theta\left(\pi, \pi^{\prime}\right) \sim 2^{-k}}} \chi_{P_{\pi}} \chi_{P_{\pi^{\prime}}}\right\|_{5 / 6}^{5 / 6} \lesssim 2^{-2 k} \delta^{-1 / 3}
$$

for all $k$ less than or equal to $\left\lfloor\log _{2}(1 / \delta)\right\rfloor$, and each ball $B_{j}$.

Without loss of generality, we can suppose that $B_{j}$ is centered on the $x_{1} x_{2}-$ plane. Define the dilation $L: \mathbb{R}^{4} \rightarrow \mathbb{R}^{4}$ by

$$
L\left(x_{1}, x_{2}, x_{3}, x_{4}\right)=\left(x_{1}, x_{2}, 2^{k} x_{3}, 2^{k} x_{4}\right) .
$$

As det $L=2^{2 k}$, if we scale our geometric configuration by $L$, then

$$
\left\|\sum_{\substack{\pi, \pi^{\prime} \in B_{j} \cap \Pi \\ \theta\left(\pi, \pi^{\prime}\right) \sim 2^{-k}}} \chi_{P_{\pi}} \chi_{P_{\pi^{\prime}}}\right\|_{5 / 6}^{5 / 6}=2^{-2 k}\left\|\sum_{\substack{\pi, \pi^{\prime} \in B_{j} \cap \Pi \\ \theta\left(\pi, \pi^{\prime}\right) \sim 2^{-k}}} \chi_{L\left(P_{\pi}\right)} \chi_{L\left(P_{\pi^{\prime}}\right)}\right\|_{5 / 6}^{5 / 6} .
$$


Essentially we have changed $\delta$ to $2^{k} \delta$, and $\theta\left(L(\pi), L\left(\pi^{\prime}\right)\right) \sim 1$, so that if we can prove (6) when $k=0$, then

$$
\left\|\sum_{\substack{\pi, \pi^{\prime} \in B_{j} \cap \Pi \\ \theta\left(\pi, \pi^{\prime}\right) \sim 2^{-k}}} \chi_{L\left(P_{\pi}\right)} \chi_{L\left(P_{\pi^{\prime}}\right)}\right\|_{5 / 6}^{5 / 6} \leq C_{\epsilon}\left(2^{k} \delta\right)^{-1 / 3-\epsilon}<C_{\epsilon} \delta^{-1 / 3-\epsilon} \lesssim \delta^{-1 / 3} .
$$

Now by combining (7) and (8),

$$
\left\|\sum_{\substack{\pi, \pi^{\prime} \in B_{j} \cap \Pi \\ \theta\left(\pi, \pi^{\prime}\right) \sim 2^{-k}}} \chi_{P_{\pi}} \chi_{P_{\pi^{\prime}}}\right\|_{5 / 6} \lesssim 2^{-2 k} \delta^{-1 / 3}
$$

so it will suffice to prove $(6)$ when $k=0$.

Now as

$$
\sum_{\pi, \pi^{\prime}} \chi_{P_{\pi}} \chi_{P_{\pi^{\prime}}} \leq \sum_{k=0}^{\left\lfloor\log _{2}(1 / \delta)\right\rfloor} \sum_{\pi, \pi^{\prime}: \underline{\theta}\left(\pi, \pi^{\prime}\right) \sim 2^{-k}} \chi_{P_{\pi}} \chi_{P_{\pi^{\prime}}}+\sum_{\pi, \pi^{\prime}: \underline{\theta}\left(\pi, \pi^{\prime}\right)<\delta} \chi_{P_{\pi}} \chi_{P_{\pi^{\prime}}},
$$

by the triangle inequality, it will suffice to show

$$
\left\|\sum_{\substack{\theta\left(\pi, \pi^{\prime}\right) \sim 1 \\ \underline{\theta}\left(\pi, \pi^{\prime}\right) \sim 2^{-k}}} \chi_{P_{\pi}} \chi_{P_{\pi^{\prime}}}\right\|_{5 / 6}^{5 / 6} \lesssim \delta^{-1 / 3}
$$

for all $k$ less than or equal to $\left\lfloor\log _{2}(1 / \delta)\right\rfloor$, and

$$
\left\|\sum_{\substack{\theta\left(\pi, \pi^{\prime}\right) \sim 1 \\ \underline{\theta}\left(\pi, \pi^{\prime}\right)<\delta}} \chi_{P_{\pi}} \chi_{P_{\pi^{\prime}}}\right\|_{5 / 6}^{5 / 6} \lesssim \delta^{-1 / 3}
$$

In (9) the planes are only intersecting in points, and in (10) the planes are almost intersecting in lines.

To prove (9), we fix $k$ and define $F$ by

$$
F=\left\{x \in \mathbb{R}^{4}: \sum_{\pi, \pi^{\prime} \in \Pi:(*)} \chi_{P_{\pi}} \chi_{P_{\pi^{\prime}}}(x) \sim \lambda\right\},
$$

where $(*)$ denotes the conditions $\theta\left(\pi, \pi^{\prime}\right) \sim 1$ and $\underline{\theta}\left(\pi, \pi^{\prime}\right) \sim 2^{-k}$. We have

$$
\left\|\sum_{\pi, \pi^{\prime} \in \Pi:(*)} \chi_{P_{\pi}} \chi_{P_{\pi^{\prime}}}\right\|_{5 / 6}^{5 / 6} \lesssim \sum_{\lambda} \lambda^{5 / 6}|F|,
$$

where $\lambda$ ranges dyadically up to a constant multiple of $\delta^{-4}$. Thus, by the triangle inequality, it will suffice to show the weak type inequality

$$
\lambda^{5 / 6}|F| \lesssim \delta^{-1 / 3} \text {. }
$$

We can assume that $|F|$ is greater than $\delta^{3}$, as otherwise we are done.

Define $\Pi_{\nu}$ by

$$
\Pi_{\nu}=\left\{\pi \in \Pi:\left|P_{\pi} \cap F\right| \sim \nu\left|P_{\pi}\right|\right\}
$$

We will use the pigeonhole principle to find a single plate that intersects many other plates that have density $\nu \gtrsim \sqrt{\lambda}|F|$. 
By definition,

$$
\int_{F} \sum_{\pi, \pi^{\prime} \in \Pi:(*)} \chi_{P_{\pi}} \chi_{P_{\pi^{\prime}}} \gtrsim \lambda|F|
$$

so that

$$
\sum_{\nu, \nu^{\prime}} \sum_{\pi \in \Pi_{\nu}} \sum_{\pi^{\prime} \in \Pi_{\nu^{\prime}}:(*)}\left|P_{\pi} \cap P_{\pi^{\prime}} \cap F\right|=\int_{F} \sum_{\pi, \pi \in \Pi:(*)} \chi_{P_{\pi}} \chi_{P_{\pi^{\prime}}} \gtrsim \lambda|F| \gtrsim \delta^{3},
$$

where the sums over $\nu$ and $\nu^{\prime}$ range dyadically from zero to one. The summands where $\nu$ or $\nu^{\prime}$ is less than a large power of $\delta$ can be absorbed by the larger summands. Thus, by the pigeonhole principle, we can find $\nu$ and $\nu^{\prime}$, which we now fix, such that

$$
\int_{F} \sum_{\pi \in \Pi_{\nu}} \sum_{\pi^{\prime} \in \Pi_{\nu^{\prime}}:(*)} \chi_{P_{\pi}} \chi_{P_{\pi^{\prime}}} \gtrsim \lambda|F|
$$

Let

$$
F^{\prime}=\left\{x \in F: \sum_{\pi \in \Pi_{\nu}} \sum_{\pi^{\prime} \in \Pi_{\nu^{\prime}}:(*)} \chi_{P_{\pi}} \chi_{P_{\pi^{\prime}}} \gtrsim \lambda\right\}
$$

so that by (12) and the pigeonhole principle,

$$
\int_{F^{\prime}} \sum_{\pi \in \Pi_{\nu}} \sum_{\pi^{\prime} \in \Pi_{\nu^{\prime}}:(*)} \chi_{P_{\pi}} \chi_{P_{\pi^{\prime}}} \gtrsim \lambda|F| \text {. }
$$

By definition,

$$
\sum_{\pi \in \Pi_{\nu}} \sum_{\pi^{\prime} \in \Pi_{\nu^{\prime}}:(*)} \chi_{P_{\pi}} \chi_{P_{\pi^{\prime}}} \leq \lambda
$$

on $F$, so that $\left|F^{\prime}\right| \gtrsim|F|$. Now as

$$
\sum_{\pi \in \Pi_{\nu}} \sum_{\pi^{\prime} \in \Pi_{\nu^{\prime}}:(*)} \chi_{P_{\pi}} \chi_{P_{\pi^{\prime}}} \gtrsim \lambda
$$

on $F^{\prime}$, we can suppose that

$$
\sum_{\pi \in \Pi_{\nu}} \chi_{P_{\pi}} \gtrsim \sqrt{\lambda}
$$

on some $F^{\prime \prime} \subset F^{\prime}$, where $\left|F^{\prime \prime}\right| \gtrsim\left|F^{\prime}\right|$. Thus

$$
\int_{F} \sum_{\pi \in \Pi_{\nu}} \chi_{P_{\pi}} \geq \int_{F^{\prime \prime}} \sum_{\pi \in \Pi_{\nu}} \chi_{P_{\pi}} \gtrsim \sqrt{\lambda}\left|F^{\prime \prime}\right| \gtrsim \sqrt{\lambda}|F| .
$$

By definition, $\nu \delta^{2} \gtrsim\left|P_{\pi} \cap F\right|$ for all $\pi \in \Pi_{\nu}$, so that

$$
\sum_{\pi \in \Pi_{\nu}} \nu \delta^{2} \gtrsim \sum_{\pi \in \Pi_{\nu}}\left|P_{\pi} \cap F\right|=\int_{F} \sum_{\pi \in \Pi_{\nu}} \chi_{P_{\pi}} \gtrsim \sqrt{\lambda}|F| .
$$

Now as $\# \Pi_{\nu} \lesssim \delta^{-2}$, we have $\nu \gtrsim \sqrt{\lambda}|F|$.

From (12), we also have

$$
\sum_{\pi^{\prime} \in \Pi_{\nu^{\prime}}} \int_{P_{\pi^{\prime}}} \sum_{\pi \in \Pi_{\nu}:(*)} \chi_{P_{\pi}} \gtrsim \lambda|F|
$$


so that, there exists a $\pi_{0} \in \Pi_{\nu^{\prime}}$, such that

$$
\int_{P_{\pi_{0}}} \sum_{\pi \in \Pi_{\nu}:(*)} \chi_{P_{\pi}} \gtrsim \delta^{2} \lambda|F|,
$$

where $(*)$ denotes the conditions $\theta\left(\pi, \pi_{0}\right) \sim 1$ and $\underline{\theta}\left(\pi, \pi_{0}\right) \sim 2^{-k}$. By Lemma 5 ,

$$
\int_{P_{\pi_{0}}} \chi_{P_{\pi}}=\left|P_{\pi} \cap P_{\pi_{0}}\right| \lesssim 2^{k} \delta^{4},
$$

so that if we define $\mathcal{P}$ by

$$
\mathcal{P}=\left\{\pi \in \Pi_{\nu}: \theta\left(\pi, \pi_{0}\right) \sim 1, \underline{\theta}\left(\pi, \pi_{0}\right) \sim 2^{-k}, P_{\pi} \cap P_{\pi_{0}} \neq \emptyset\right\},
$$

then by (13), we have

$$
\# \mathcal{P} \gtrsim 2^{-k} \delta^{-2} \lambda|F|
$$

Thus we have a large set of planes, with density $\nu \gtrsim \sqrt{\lambda}|F|$, that intersect $\pi_{0}$.

As $\theta\left(\pi, \pi_{0}\right) \sim 1$ for all $\pi \in \mathcal{P}$, we can define $\Sigma=\left\{x \in \mathbb{R}^{4}: d\left(x, S_{\pi_{0}}\right)>\nu / C\right\}$ for some sufficiently large constant $C$, so that

$$
\int_{F} \chi_{P_{\pi} \cap \Sigma} \gtrsim \nu \delta^{2} \text {. }
$$

Summing over $\mathcal{P}$, we have

$$
\int_{F} \sum_{\pi \in \mathcal{P}} \chi_{P_{\pi} \cap \Sigma} \gtrsim \nu \delta^{2} \# \mathcal{P},
$$

so that by the Cauchy-Schwarz inequality,

$$
\left\|\sum_{\pi \in \mathcal{P}} \chi_{P_{\pi} \cap \Sigma}\right\|_{2} \gtrsim \frac{\nu \delta^{2} \# \mathcal{P}}{|F|^{1 / 2}} .
$$

We will use the geometry of the construction to bound the left hand side of (15) from above, in order to obtain (11). If we fix a $\pi_{1} \in \mathcal{P}$, then by Lemma 6 , there exists a 3 -space $\Lambda$, such that

$$
\min _{\pi \subset \Lambda} \theta\left(\pi, \pi_{1}\right)<2^{-k}
$$

and if $\pi_{2} \in \mathcal{P}$ and $P_{\pi_{1}} \cap P_{\pi_{2}} \cap \Sigma \neq \emptyset$, then

$$
\pi_{2} \in \mathcal{M}_{\Lambda}^{\nu^{-1} 2^{-k}}
$$

where $\mathcal{M}_{\Lambda}^{\nu^{-1} 2^{-k}}=\left\{\pi^{\prime} \in \mathcal{M}: \min _{\pi \subset \Lambda} \theta\left(\pi, \pi^{\prime}\right)<\nu^{-1} 2^{-k}\right\}$.

Now, as $\mathcal{M}$ is curved, there exists a constant $C$ such that

$$
\frac{\mu\left(\mathcal{M}_{\Lambda}^{\epsilon_{1}} \cap B_{\epsilon_{2}}(\pi)\right)}{\epsilon_{1} \epsilon_{2}}<C
$$

for all $\epsilon_{1}, \epsilon_{2}>0,3$-spaces $\Lambda$, and $\pi \in \mathcal{M}$. Thus, as $\mathcal{P}$ is $\delta$-separated, we see that

$$
\#\left\{\pi_{2} \in \mathcal{P}: \theta\left(\pi_{1}, \pi_{2}\right) \leq 2^{-j}, \quad P_{\pi_{1}} \cap P_{\pi_{2}} \cap \Sigma \neq \emptyset\right\} \lesssim \nu^{-1} 2^{-k} 2^{-j} \delta^{-2},
$$

where we take $j$ to be less than or equal to $\left\lfloor\log _{2}(1 / \delta)\right\rfloor$. By Lemma 4,

$$
\left|P_{\pi_{1}} \cap P_{\pi_{2}} \cap \Sigma\right| \lesssim 2^{j} \delta^{3}
$$


when $\theta\left(\pi_{1}, \pi_{2}\right) \sim 2^{-j}$, so that

$$
\sum_{\pi_{2} \in \mathcal{P}: \theta\left(\pi_{1}, \pi_{2}\right) \sim 2^{-j}}\left|P_{\pi_{1}} \cap P_{\pi_{2}} \cap \Sigma\right| \lesssim \nu^{-1} 2^{-k} \delta .
$$

Hence, by the triangle inequality,

$$
\sum_{\pi_{2} \in \mathcal{P}}\left|P_{\pi_{1}} \cap P_{\pi_{2}} \cap \Sigma\right| \lesssim \nu^{-1} 2^{-k} \delta
$$

so that by summing over $\mathcal{P}$, we have

$$
\sum_{\pi_{1} \in \mathcal{P}} \sum_{\pi_{2} \in \mathcal{P}}\left|P_{\pi_{1}} \cap P_{\pi_{2}} \cap \Sigma\right| \lesssim \nu^{-1} 2^{-k} \delta \# \mathcal{P} .
$$

Now

$$
\left\|\sum_{\pi \in \mathcal{P}} \chi_{P_{\pi} \cap \Sigma}\right\|_{2}^{2}=\sum_{\pi_{1} \in \mathcal{P}} \sum_{\pi_{2} \in \mathcal{P}}\left|P_{\pi_{1}} \cap P_{\pi_{2}} \cap \Sigma\right|
$$

so that

$$
\left\|\sum_{\pi \in \mathcal{P}} \chi_{P_{\pi} \cap \Sigma}\right\|_{2} \lesssim\left(\nu^{-1} 2^{-k} \delta \# \mathcal{P}\right)^{1 / 2}
$$

Combining the equations (15) and (18), and the fact that $\# \mathcal{P} \gtrsim 2^{-k} \delta^{-2} \lambda|F|$, we obtain

$$
\nu^{3} \lambda \lesssim \delta^{-1}
$$

Using the fact that $\nu \gtrsim \sqrt{\lambda}|F|$, we have

$$
\lambda^{5 / 2}|F|^{3} \lesssim \delta^{-1}
$$

and we take the third root to obtain (11) as required.

To prove $(10)$, we argue in the same way. We let $(*)$ denote the conditions $\theta\left(\pi, \pi^{\prime}\right) \sim 1$ and $\underline{\theta}\left(\pi, \pi^{\prime}\right)<\delta$. We apply Lemma 4 in place of Lemma 5 , so that the estimate (14) becomes

$$
\int_{P_{\pi_{0}}} \chi_{P_{\pi}}=\left|P_{\pi} \cap P_{\pi_{0}}\right| \lesssim \delta^{3},
$$

and $\# \mathcal{P} \gtrsim \delta^{-1} \lambda|F|$. The expressiones in (16) and (17) are changed to

$$
\min _{\pi \subset \Lambda} \theta\left(\pi, \pi_{1}\right)<\delta \quad \text { and } \quad \pi_{2} \in \mathcal{M}_{\Lambda}^{\nu^{-1} \delta}
$$

so that

$$
\left\|\sum_{\pi \in \mathcal{P}} \chi_{P_{\pi} \cap \Sigma}\right\|_{2} \lesssim\left(\nu^{-1} \delta^{2} \# \mathcal{P}\right)^{1 / 2} .
$$

As before, we combine these equations with (15), which is unchanged, so that

$$
\lambda^{5 / 2}|F|^{3} \lesssim \delta^{-1}
$$

and we are done. 


\section{Proof of Theorems 1 and 2}

The following argument is well known, and can be found in [2]. Let $\left\{B\left(x_{j}, r_{j}\right)\right\}$ be a covering of a $\mathcal{K}_{2}^{n}$-set $E$, where $r_{j} \leq 1 / 4$. We are required to show

$$
\sum_{j} r_{j}^{\frac{n+3}{2}-\epsilon} \geq C_{\epsilon}>0
$$

for all $\epsilon>0$.

Define $E_{k}$ by

$$
E_{k}=E \cap \bigcup_{r_{j} \sim 2^{-k}} B\left(x_{j}, r_{j}\right)
$$

for $k \geq 2$. As $E$ is a $\mathcal{K}_{2}^{n}$-set, for all $\pi \in \mathcal{M}$ there is a square $S_{\pi} \subset E$ with a corresponding plate $P_{\pi}$. By the pigeonhole principle, for all $\pi \in \mathcal{M}$ there is a $k_{\pi}$ such that

$$
\mathcal{L}^{2}\left(S_{\pi} \cap E_{k_{\pi}}\right) \geq \frac{1}{k_{\pi}^{2}} \gtrsim 1
$$

Thus $\mathcal{M}=\bigcup_{k \geq 2} \mathcal{M}_{k}$, where

$$
\mathcal{M}_{k}=\left\{\pi \in \mathcal{M}: \mathcal{L}^{2}\left(S_{\pi} \cap E_{k}\right) \geq \frac{1}{k^{2}}\right\}
$$

By the pigeonhole principle again, there exists a $k_{0}$ such that

$$
\mu\left(\mathcal{M}_{k_{0}}\right) \geq \frac{1}{k_{0}^{2}} \gtrsim 1
$$

where $\mu$ is the induced measure on $\mathcal{M}$, normalised to have total mass 1 .

Let $\delta=2^{-k_{0}}$, and let $\Pi$ be a maximal $\delta$-separated subset of $\mathcal{M}_{k_{0}}$, so that $\# \Pi \gtrsim \delta^{2-n}$. Define $J=\left\{j: r_{j} \sim \delta\right\}$ and $E_{\delta}=\bigcup_{j \in J} B\left(x_{j}, 2 \delta\right)$, so that

$$
\int_{E_{\delta}} \chi_{P_{\pi}} \gtrsim \delta^{n-2} \mathcal{L}^{2}\left(S_{\pi} \cap E_{k_{0}}\right) \geq \frac{\delta^{n-2}}{k_{0}^{2}} \gtrsim \delta^{n-2} .
$$

Now as $\# \Pi \gtrsim \delta^{2-n}$, we have

$$
\sum_{\pi \in \Pi} \int_{E_{\delta}} \chi_{P_{\pi}} \gtrsim 1
$$

so that by Hölder's inequality,

$$
\left|E_{\delta}\right|^{\frac{2}{n+1}}\left\|\sum_{\pi \in \Pi} \chi_{P_{\pi}}\right\|_{\frac{n+1}{n-1}} \gtrsim 1
$$

By Lemmas 7 and 8, we have

$$
\left\|\sum_{\pi \in \Pi} \chi_{P_{\pi}}\right\|_{\frac{n+1}{n-1}} \lesssim \delta^{\frac{3-n}{n+1}}
$$

where $n=3$ or 4 , so that

$$
\left|E_{\delta}\right| \gtrsim \delta^{\frac{n-3}{2}} .
$$

On the other hand, we have $\# J \delta^{n} \gtrsim\left|E_{\delta}\right|$, so that

$$
\# J \geq C_{\epsilon} \delta^{-\frac{n+3}{2}+\epsilon}
$$


for all $\epsilon>0$. Hence, when $n=3$ or 4 ,

$$
\sum_{j} r_{j}^{\frac{n+3}{2}-\epsilon} \geq \# J\left(\frac{\delta}{2}\right)^{\frac{n+3}{2}-\epsilon} \geq C_{\epsilon}^{\prime}>0
$$

for all $\epsilon>0$, and we are done.

\section{Sharpness in the complex case}

We construct an example, inspired by the Heisenberg group example in [6], to show that Theorem 3 is sharp. Define $E \subset \mathbb{C}^{4}$ by

$$
E=\left\{\left(z_{1}, z_{2}, z_{3}, z_{4}\right) \in \mathbb{C}^{4}: \operatorname{Im}\left(z_{1} \overline{z_{2}}\right)=\operatorname{Im}\left(z_{3} \overline{z_{4}}\right)\right\},
$$

so that $\operatorname{dim}_{H}(E)=7$. Define the planes $\pi_{u, v}$ by

$$
\pi_{u, v}=\left\{(1,-|v| \operatorname{Im}(u) i, u,|v|) z+\left(1,|v| \operatorname{Im}(u) i, \frac{\bar{u} v}{|v|}, v\right) z^{\prime}: z, z^{\prime} \in \mathbb{C}\right\},
$$

and the manifold $\mathcal{M}$ by

$$
\mathcal{M}=\left\{\pi_{u, v}: u, v \in \mathbb{C}, \operatorname{Im}(u) \neq 0, \operatorname{Im}(v) \neq 0\right\} .
$$

It is not hard to calculate that the planes are contained in $E$, and it is clear that $\mathcal{M}$ is a 2-dimensional manifold as a subset of $\operatorname{Gr}(4,2)$ over $\mathbb{C}$.

It remains to show that $\mathcal{M}$ is curved. It will suffice to show that

$$
\{\pi \in \mathcal{M}: \pi \subset \Lambda\}
$$

is no more than 1 -dimensional for all 3 -spaces $\Lambda$. Now if a plane in $\mathcal{M}$ is contained in a 3 -space $\Lambda$, then

$$
(1,-|v| \operatorname{Im}(u) i, u,|v|) \cdot\left(a_{1}, a_{2}, a_{3}, a_{4}\right)=a_{1}-a_{2}|v| \operatorname{Im}(u) i+a_{3} u+a_{4}|v|=0
$$

and

$$
\left(1,|v| \operatorname{Im}(u) i, \frac{\bar{u} v}{|v|}, v\right) \cdot\left(a_{1}, a_{2}, a_{3}, a_{4}\right)=a_{1}+a_{2}|v| \operatorname{Im}(u) i+a_{3} \frac{\bar{u} v}{|v|}+a_{4} v=0
$$

for some normal $\left(a_{1}, a_{2}, a_{3}, a_{4}\right) \in \mathbb{C}^{4}$. We can multiply the second equation by $|v| / v$ and subtract it from the first to solve for $\operatorname{Im}(u)$ in terms of $v$. Substituting back into the first equation, we fix $u$ in terms of $v$, so that the set of planes contained in $\Lambda$ is parametrized by a single variable. Thus the restriction of $\mathcal{M}$ to a 3 -space is no more than 1 -dimensional, and $E$ is a $\mathcal{K}_{2}^{4}$-set.

Thus, in the complex case, the curvature condition is not sufficient to guarantee nontrivial intersection, even before translation. This example does not extend to the reals (or the finite fields, as there is no square root), and the curvature condition is stronger over $\mathbb{R}$ than over $\mathbb{C}$. It seems possible that the real and complex cases are qualitatively different. Thus the problem of sharp lower bounds for the Hausdorff dimension of real $\mathcal{K}_{2}^{n}$-sets is open and interesting for $n \geq 4$. 


\section{Final remarks}

We could define the $\mathcal{K}_{2}^{n}$-sets so that they only contain a unit square parallel to each direction plane, and not necessarily the whole plane.

Theorems 1 and 2 would extend in a natural way to $\mathcal{K}_{k}^{n}$-sets, where $k=n-1$ or $n-2$, and the planes are replaced by $k$-planes. We note that the $\mathcal{K}_{2}^{4}$-set over $\mathbb{C}$ of the previous section may not be a $\mathcal{K}_{4}^{8}$-set over $\mathbb{R}$, as the curvature condition is stronger over $\mathbb{R}$.

It also seems likely that we could adapt the proofs to obtain the corresponding maximal function estimates. We neglect these potential generalizations mainly for expository purposes.

\section{Acknowledgements}

Many thanks to Gerd Mockenhaupt for suggesting a similar planar variant problem which led to the consideration of this one. Thanks to Peter Sjögren, Thomas Duyckaerts, Fulvio Ricci, Ana Vargas and Toby Bailey for helpful conversations, and thanks to an anonymous referee for pointing out a mistake in an earlier version.

\section{References}

[1] A. Besicovitch, On Kakeya's problem and a similar one, Mat. Zeit. 27 (1928) 312-320.

[2] J. Bourgain, Besicovitch type maximal operators and applications to Fourier analysis, Geom. Funct. Anal. 1 (1991), no. 2, 147-187.

[3] On the dimension of Kakeya sets and related maximal inequalities, Geom. Funct. Anal. 9 (1999), no. 2, 256-282.

[4] A. Córdoba, The Kakeya maximal function and the spherical summation multipliers, Amer. J. Math. 99 (1977), no. 1, 1-22.

[5] N. H. Katz and T. Tao, Bounds on arithmetic projections, and applications to the Kakeya conjecture, Math. Res. Lett. 6 (1999), no. 5-6, 625-630.

[6] N. H. Katz, I. Łaba and T. Tao, An improved bound on the Minkowski dimension of Besicovitch sets in $\mathbf{R}^{3}$, Ann. of Math.(2) 152 (2000), no. 2, 383-446.

[7] _ New bounds for Kakeya problems, J. Anal. Math. 87 (2002) 231-263.

[8] I. Łaba, T. Tao, An improved bound for the Minkowski dimension of Besicovitch sets in medium dimension, to appear.

[9] T. Mitsis, (n,2)-sets have full Hausdorff dimension, Rev. Mat. Iberoamericana, 20 (2004), no. 2, 381-393.

[10] G. Mockenhaupt and T. Tao, Restriction and Kakeya phenomena for finite fields, Duke Math. J. 121 (2004), no. 1, 35-74.

[11] T. Tao, A new bound for finite field Besicovitch sets in four dimensions, to appear in Pacific J. Math.

[12] - Restriction theorems and applications, available at www.math.ucla.edu/ ${ }^{\text {tao. }}$

[13] T. Tao, A. Vargas and L. Vega, A bilinear approach to the restriction and Kakeya conjectures, J. Amer. Math. Soc. 11 (1998), no. 4, 967-1000.

[14] T. Wolff, An improved bound for Kakeya type maximal functions, Rev. Mat. Iberoamericana 11 (1995), no. 3, 651-674.

Department of Mathematics, Universidad Autónoma de Madrid, 28049 Madrid, SPAIN

E-mail address: K.M.Rogers.99@cantab.net 